\title{
Pharmacoresistant Abdominal Seizures in Symptomatic Localization-Related Epilepsy
}

\section{Case Report}

Journal of Epilepsy Research pISSN 2233-6249 / eISSN 2233-6257

Received May 17, 2021

Revised June 3, 2021

Accepted June 8, 2021

Corresponding author:

Sachin Sureshbabu, MD, DM

Sr consultant Neurologist and Epileptologist

Department of Neurology, Aster MIMS

Hospital, Aster MIMS, Kozhikode, Kerala

673016, India

Tel. $+919207028028,+918375938480$

Fax. +91-4952741329

E-mail; drsachins1@rediffmail.com

\author{
Vadakke Puthanveetil Tushar, MD, DM, Sachin Sureshbabu, MD, DM, \\ Kunnath Gopalakrishnan Sruthi, BSC, Smilu Mohanlal, MD
}

Department of Neurology, Aster MIMS Hospital, Kerala, India

\begin{abstract}
Abdominal epilepsy is an uncommon cause of recurrent abdominal pain with or without other complaints seen in children and adults which often goes unnoticed. Here we are presenting a case of abdominal epilepsy in a 7-year boy who had recurrent abdominal pain since many years. He had a history of ventriculoperitoneal shunting which was performed for obstructive hydrocephalus at 1 month and left hemiparesis. He was evaluated at multiple centers for abdominal complaints before being referred here. The video electroencephalogram done showed inter ictal and ictal abnormalities which correlated with magnetic resonance imaging and positron emission tomography abnormalities on the right side. He was started on antiepileptics with a partial response and continued to get events even with four antiepileptics but the frequency and severity reduced significantly. The present case report is to highlight the drug refractoriness of abdominal seizures in a patient with symptomatic focal epilepsy. (2021;11:106-109)
\end{abstract}

Key words: Focal epilepsy, Abdominal epilepsy, Pharmacoresistant

\section{Introduction}

Abdominal epilepsy (AE) is an uncommon cause of recurrent abdominal pain with or without other non-specific abdominal complaints like nausea or vomiting. It can be seen in children and adults. Because of the non-specific nature of complaints, it often goes undiagnosed or mismanaged, and thus should be thought of in all cases of recurrent or intractable abdominal complaints not responding to standard medical management. ${ }^{1}$

\section{Case Report}

A 7-year-old right-handed boy, first in birth order, born to non-consanguineously married couple, presented with the complaints of recurrent abdominal pain. He was born by vacuum assisted vaginal delivery with no immediate neonatal complications. However, on 27th day of life, he was diagnosed to have hydrocephalus and underwent a right parietal Codman Bactiseal ventriculoperitoneal (VP) shunt. Post VP shunt, parents noticed paucity of left upper and lower limb movements. He had delayed motor milestones wherein he started walking by 3 years and speech was attained at 2 years. From 4 years of age, he started complaining of momentary abnormal epigastric sensations, lasting for 1-2 seconds and occurring rarely (once in 3-6 months), which were treated as gastritis with proton pump inhibitors. He did not have any visual symptoms. At the age of 5 , he had fever-triggered clonic movements of left lower face and left upper and lower limb that lasted for 30 minutes (fever-triggered focal status epilepticus), for which he was initiated on carbamazepine. He continued to have recurrent episodes of abnormal epigastric sensations in the form of an abrupt onset sharp catching pain causing considerable discomfort. It was not associated with other autonomic manifestations like vomiting, coughing, hypersalivation, perioral pallor, diaphoresis or piloerection. The frequency of episodes increased to 1 to 2 times per month prompting a revaluation by the gastro-enterologist and the pediatrician before being finally referred for evaluation to our center.

In view of clinical suspicion of seizures, a video electroencephalogram (EEG) was done that showed (Fig. 1) interictal epileptiform abnormalities in the form of spike and slow wave discharges in right posterior head region $(\mathrm{T} 6, \mathrm{P} 4, \mathrm{O} 2)$ and intermittent polymorphic delta activity over the right frontotemporal region. During the recording, he had multiple clinical events (abdominal pain \pm retching) with the EEG showing a low voltage fast activity over C4, P4 followed by a delta rhythm in right temporal region, which later culminates in a 2-3 $\mathrm{Hz}$ repetitive spiking in right posterior head regions. His magnetic 
A

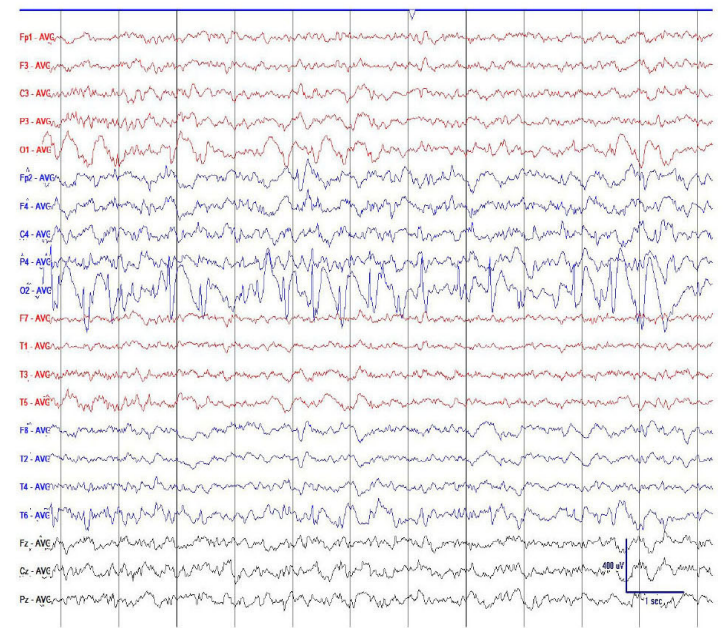

B

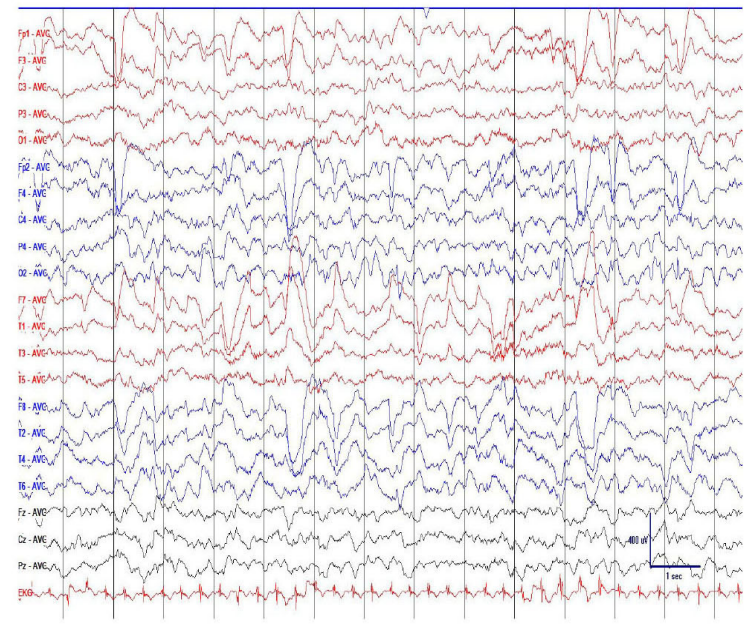

Figure 1. (A) Interictal electroencephalogram (EEG) showing right posterior head region (predominantly occipital spike). (B) Ictal EEG shows polymorphic delta rhythm in the temporal and posterior head region on the right hemisphere.
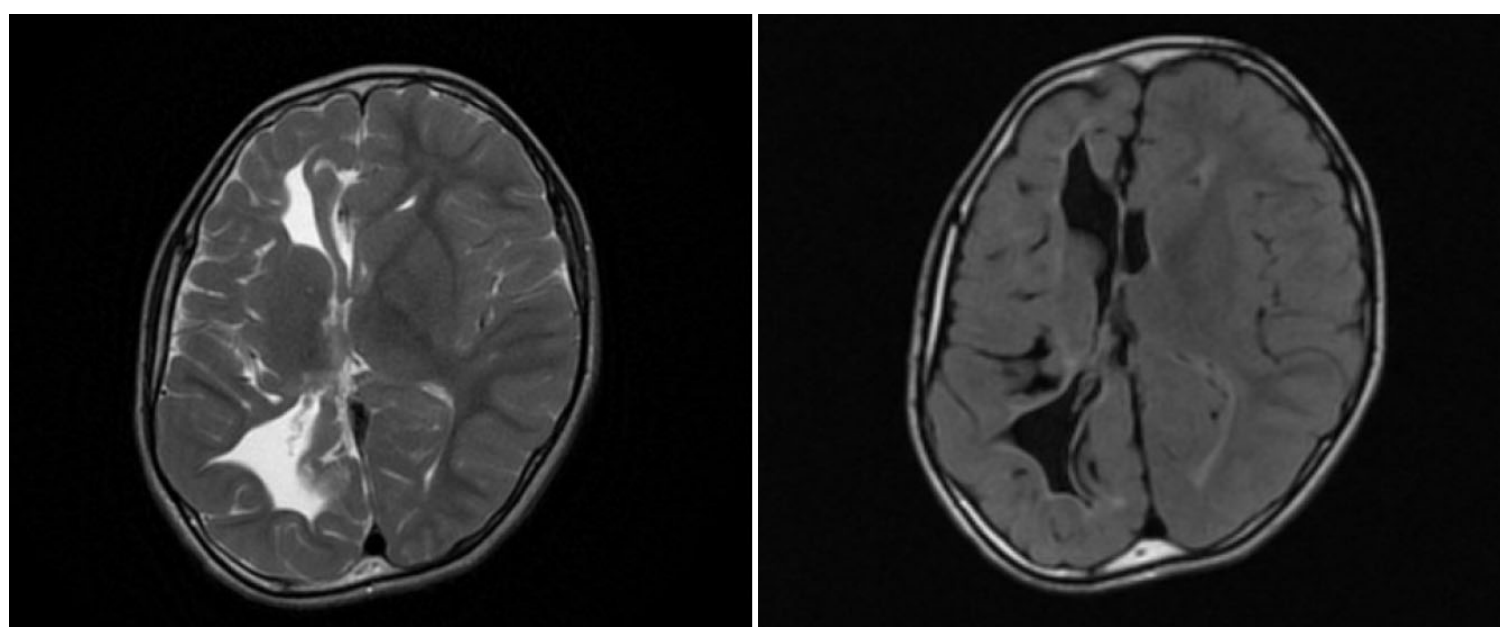

Figure 2. T2 weighted and fluid-attenuated inversion recovery (FLAIR) images shows a holohemispheric global atrophy of right cerebral hemisphere with thinned out corpus callosum and exvacuo dilatation of the ipsilateral ventricle with slight shift of midline structures to right.

resonance imaging (MRI) brain showed (Fig. 2) hemi atrophy, poor grey-white differentiation, white matter hyperintensities and paucity of white matter. Fluro-deoxy glucose positron emission tomography (PET) scan of the brain (Fig. 3) showed hypermetabolism in the occipital region, which could correspond to the high spike load on interictal EEG. He presently has 1 to 2 episodes per week on perampanel, zonisamide, oxcarbazepine and clobazam. The regime was changed a couple of weeks back only.

\section{Discussion}

AE is a term applied to a generalized or focal epilepsy where abdominal symptoms are the dominant feature of the clinical seizure. It can be in the form of abdominal pain, discomfort, nausea, vomiting, bloating sensation or diarrhea. ${ }^{1}$ These symptoms can be due to peristaltic contractions or activation of the visceroceptive sensory apparatus. ${ }^{2}$ The distribution of the abdominal pain can be epigastric, peri-umbilical or diffuse. ${ }^{3}$ Symptoms are paroxysmal lasting from few seconds to an hour, which may or may not be associated with other 

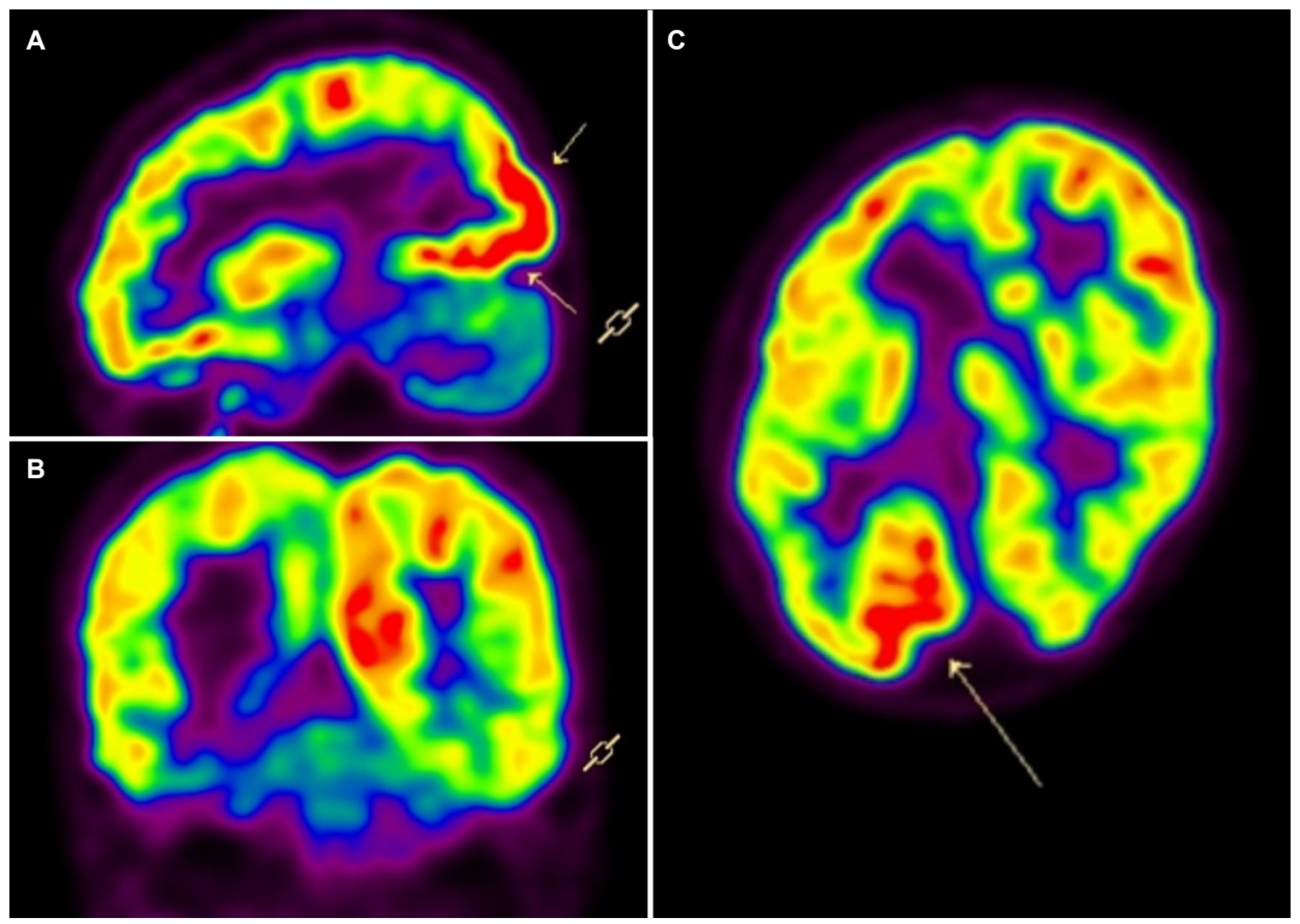

Figure 3. Fluorodeoxyglucose-positron emission tomography (FDG PET) sagittal (A), coronal (B), and axial (C) images showing generalized hypometabolism in right cerebral hemisphere except for right occipital region which shows hypermetabolism (arrows).

ictal features like behavioral arrest, automatisms, aphasia, headache, and post ictal confusion. When abdominal symptoms are the only feature, it is not unusual for the patients to land up in the gastroenterology or even the psychiatry departments. ${ }^{4}$ Associated headache can lead to a diagnosis of abdominal migraine. EEG abnormalities may clinch the diagnosis but can be absent in a vast majority of cases. $^{5}$

Abdominal symptoms have been described in both focal and generalized epilepsies. A limbic network involving temporal lobe and anterior insular or a benign epilepsy syndrome like Panayiotopoulos syndrome can manifest with predominant or exclusive abdominal symptoms. Autonomic features like sweating, pallor, pilo-erection may be seen in association. ${ }^{6}$ The presentation is more common in children, but adult cases have also been reported. The present report highlights some important and unique aspects of $A E$, which have not received emphasis before in literature: 1) AE dominated by abdominal pain (not typical rising sensation) without accompanying ictal signs occurring as the dominant seizure type in a symptomatic focal epilepsy with structural abnormalities on MRI is previously unreported. 2) Pharmacoresistance is not a usual feature of $A E$, but our patient was refractory to multiple anti-seizure medications (four of them). ${ }^{78}$ And 3) potential involvement of parieto-occipital cortex (irritative zone) is also a novel observation. ${ }^{9,10}$ Our hypothesis for the epileptogenic zone was insulo-opercular in view of the following features: 1) history of facial clonic movements, 2) abdominal pain and retching, and 3) delta rhythm at onset (indicating a deeper location of the focus). This also argues against the parieto-occipital origin which should have produced a more superficial and distinct fast rhythm or repetitive spiking.

The presence of a narrow epileptic network within a large area of dysplastic cortex raises one more pertinent question or rather possibility that is, in the event of failure with further trial of antiepileptic drug combinations, a focal resection rather than a disconnection of the hemispheres should be considered.

$A E$ is a challenging diagnosis which can be established only by careful analysis of clinical symptoms, recording of clinical events by video-telemetry and sustained response to antiepileptics. A focal epilepsy presenting with pharmacoresistant abdominal symptoms is presented to highlight the unusual phenotype with its diagnostic and 
therapeutic implications.

\section{Conflict of Interest}

The authors declare that they have no conflicts of interest.

\section{References}

1. Deswal S, Paul P, Murugan S, Yadav TP. Abdominal epilepsy- a diagnosis often missed! - a case report. Int J Pediatr Res 2018;5:598-600.

2, Sengupta JN. Visceral pain: the neurophysiological mechanism. Handb Exp Pharmacol 2009;(194):31-74.

3. Lo Bianco G, Thomson S, Vigneri S, Shapiro H, Schatman ME. Abdominal epilepsy, a rare cause of abdominal pain: the need to investigate thoroughly as opposed to making rapid attributions of psychogenic causality. J Pain Res 2020;13:457-60.
4. Harshe DG, Harshe SD, Harshe GR, Harshe GG. Abdominal epilepsy in an adult: a diagnosis often missed. J Clin Diagn Res 2016;10:VD01-2.

5. Dutta SR, Hazarika I, Chakravarty BP. Abdominal epilepsy, an uncommon cause of recurrent abdominal pain: a brief report. Gut 2007;56:439-41.

6. Koutroumanidis M. Panayiotopoulos syndrome. BMJ 2002;324:1228-9.

7. Cerminara C, El Malhany N, Roberto D, Curatolo P. Focal epilepsy with ictal abdominal pain: a case report. Ital J Pediatr 2013;39:76.

8. Kshirsagar VY, Nagarsenkar S, Ahmed M, Colaco S, Wingkar KC. Abdominal epilepsy in chronic recurrent abdominal pain. J Pediatr Neurosci 2012;7:163-6.

9. Siegel AM, Williamson PD, Roberts DW, Thadani VM, Darcey TM. Localized pain associated with seizures originating in the parietal lobe. Epilepsia 1999;40:845-55.

10. Musavi Mirzaee SM, Akbari A. Recurrent abdominal pain as a manifestation of temporal lobe epilepsy: a case report. Mod Care J 2019; 16: e90246. 